\title{
Primary Osteosarcoma of the Breast
}

\author{
Prashant Balwant Kerkar ${ }^{1} \cdot$ Garima Daga ${ }^{1}$ \\ Received: 9 July 2017 / Accepted: 13 July 2018 / Published online: 30 July 2018 \\ (C) Indian Association of Surgical Oncology 2018
}

\begin{abstract}
Extraskeletal osteosarcomas are extremely rare and account for $<1 \%$ of the soft tissue sarcomas. Most common site is in soft tissues of the lower extremities. Primary osteosarcomas of the breast are extremely rare and only a few cases have been reported in literature. It carries a very dismal prognosis in view of its high rate of local recurrence and distant spread. We report a case of primary osteosarcoma of the breast. A 44-year-old female presented with a $14 \times 10 \mathrm{~cm}$ solid mass in right breast. She underwent wide excision and frozen section was consistent with metaplastic carcinoma. A possibility of malignant phyllodes tumor was kept therefore right axillary dissection was also done along with. Final histopathology was consistent with high-grade osteogenic sarcoma in view of haphazardly arranged spindle to stellate cells with presence of giant cells and a matrix showing osteoid and cartilaginous differentiation and on IHC tumor cells were positive for Vimentin, EMA, Pankeratin, and P 63 and negative for ER, PR, Her 2 neu, CK 7, CK 8/18, and CK 34. It is imperative to recognize this rare pathologic tumor variant in view of its need of multimodal management and overall poor prognosis. Immunohistochemistry plays an important part in establishing the diagnosis and hence the further management.
\end{abstract}

Keywords Osteosarcoma $\cdot$ Breast $\cdot$ Primary

\section{Introduction}

Primary osteosarcomas of the breast are very rare, aggressive, and heterogeneous neoplasms derived from nonepithelial elements of the gland and represent less than $1 \%$ of breast cancers and less than $5 \%$ of all sarcomas [1]. They constitute a common histology after breast radiation therapy and arise mostly from adjacent bony structures and do not represent primary breast sarcomas. Histology remains indistinguishable from the conventional bone and extra skeletal osteosarcomas. Mechanism of tumorogenesis remains unclear. Unfortunately, primary breast osteosarcoma occurs in older patients [2]. It carries a poor prognosis with early tumor recurrence and a propensity for hematogenous spread most commonly to the lungs [3]. We report a case of a 44-year-old woman with a primary osteosarcoma in her right breast.

Garima Daga

narsinghbaba@yahoo.co.in

Prashant Balwant Kerkar

drprashantkerkar@hotmail.com

1 Department of Surgical Oncology, Bombay Hospital, Bombay hospital Marg, Marine Drive, Mumbai, Maharashtra 400020, India

\section{Case Report}

A 44-year-old premenopausal woman presented with two lumps in her right breast, one being for 12 years and other for 4 months. Clinical examination revealed a $14 \times 10 \mathrm{~cm}$ hard, nontender, and mobile mass, localized at 1 to 3 o'clock position in upper-inner quadrant. Another $4 \times$ $4 \mathrm{~cm}$ soft nontender mobile lump in central quadrant highly suggestive of a fibroadenoma was also palpable. Diagnostic mammography described a $14 \times 12 \mathrm{~cm}$ mass with gross central and clustered peripheral spotty calcifications (Fig. 1). No clinically enlarged nodes were detected in both axilla. High resolution ultrasonography demonstrated a densely shadowing mass, compatible with the extensive calcification identified on mammography. Doppler evaluation revealed marked vascularity in the surrounding tissue. Cytology was suggestive of metaplastic carcinoma. Staging computed tomography scan and skeletal scintigraphy showed no evidence of metastatic disease. Laboratory findings were normal, including the preoperative alkaline phosphatase. As cytology was suggestive of metaplastic carcinoma, she underwent right upper-inner quadrantectomy with complete axillary dissection. Breast reconstruction was done using latissimus dorsi flap (Fig. 2). Grossly, there 


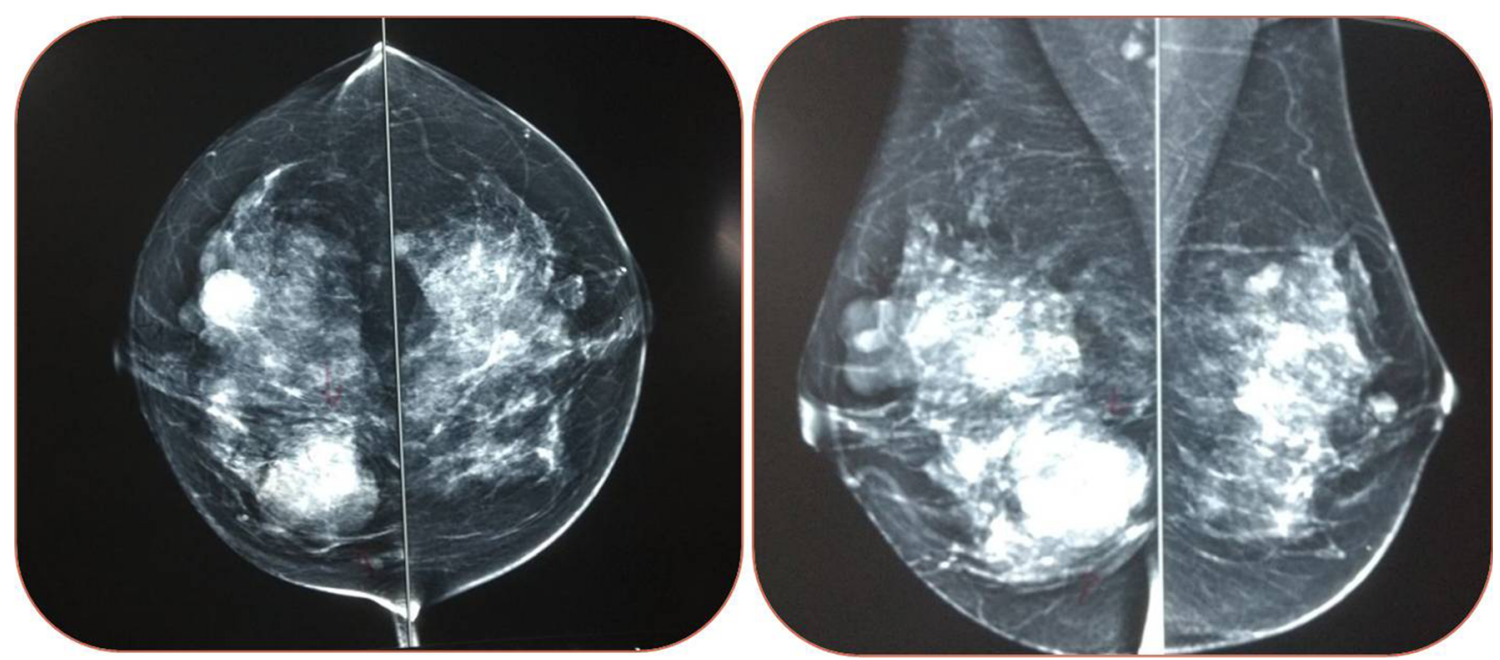

Fig. 1 Diagnostic mammogram depicting a $14 \times 12 \mathrm{~cm}$ mass with gross central and clustered peripheral spotty calcifications in right breast

were two well-circumscribed, lobulated whitish tumors of $4 \times 3 \times 2 \mathrm{~cm}$ and $14 \times 12 \times 6 \mathrm{~cm}$ size respectively with calcifications and hemorrhagic cystic areas within. Microscopically, the tumor had infiltrative borders composed of oval polyhedral, spindle to stellate cells and fusiform cells possessing enlarged, hyperchromatic, pleomorphic nuclei with osteoid, and cartilaginous matrix with severe nuclear atypia and atypical mitoses. Large vascular spaces lined by neoplastic cells and osteoclastic giant cells were present in between. The smaller lump was consistent with hyalinized fibroadenoma. All 13 axillary lymph nodes were negative for metastasis. Extensive effort was made to exclude metaplastic carcinoma. On immunohistochemistry, neoplastic cells were positive for Vimentin, p 63, EMA, and Pankeratin and negative for HMW-CK, CD-34, CK-7, ER, PR, and Her-2 neu (Fig. 3). The diagnosis of primary osteosarcoma of the breast was formulated. She received four cycles of adjuvant chemotherapy with ifosfamide and Adriamycin and currently shows no evidence of disease after 13 months of follow-up.

\section{Discussion}

Osteosarcomas of the breast are an infrequent and highly aggressive tumors with a prevalence of $<1 \%$ of all primary breast malignancies. Mean age of presentation is 65 years. Due to the rarity of the disease optimal treatment remains unclear [1]. Origin from totipotent mesenchymal cells of the breast stroma or a transformation from a pre-existing fibroadenoma or phyllodes tumors has been suggested [4]. Risk factors have been identified for extraskeletal osteosarcomas including prior local irradiation, trauma, or a foreign body. A preoperative diagnosis is unusual and only histopathology establishes the final diagnosis.

On mammography diagnosis is suggested by appearance of a large mass with well-defined margin and lobulated border with coarse or dense calcifications. A definitive diagnosis of primary can only be established when an osteogenic sarcoma arising from the underlying bones is excluded and immunohistochemical (IHC) tests show positivity for vimentin with absence of epithelial, neural, muscular, and other markers [5].
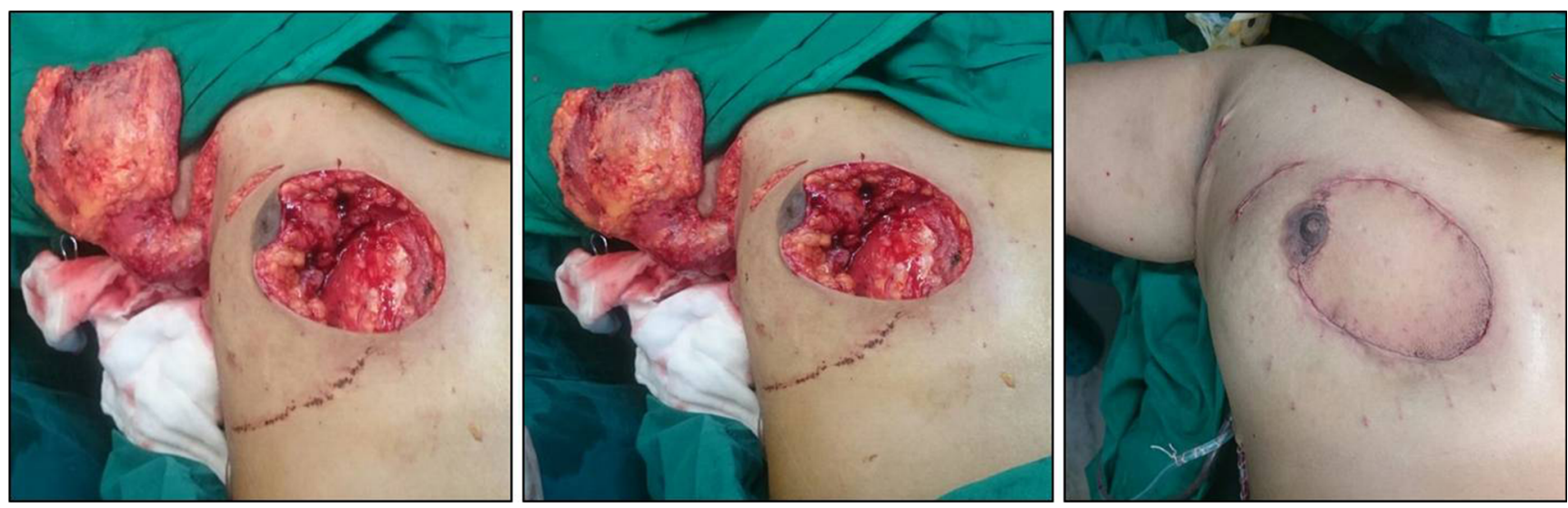

Fig. 2 Right upper-inner quadrantectomy with latissimus dorsi flap reconstruction 
Fig. 3 a $\times 400$ H\&E staining revealed oval polyhedral, spindle to stellate cells and fusiform cells possessing enlarged, hyperchromatic, pleomorphic nuclei with osteoid, and cartilaginous matrix with severe nuclear atypia and atypical mitoses. b Neoplastic cells were positive for Vimentin on IHC
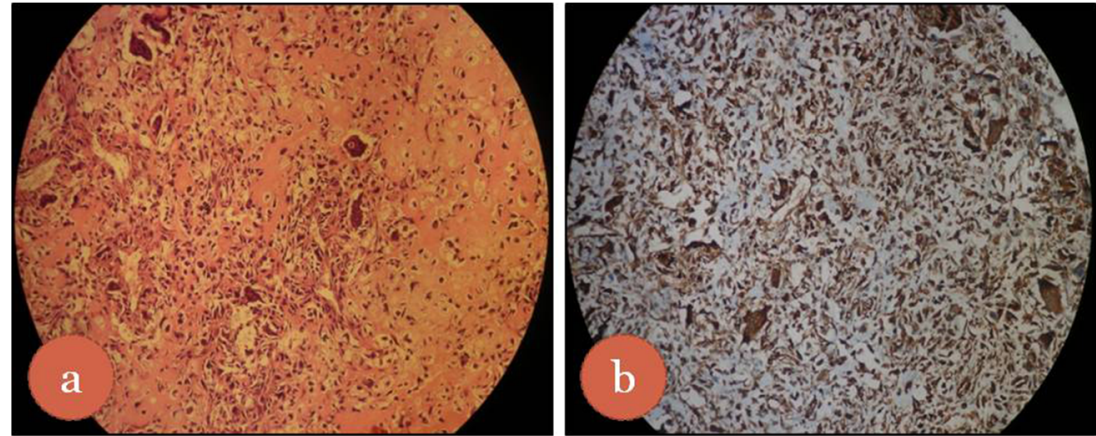

Main stay of treatment for primary osteosarcoma of breast is wide excision with negative margins. A complete resection with negative resection margins is needed, as margin involvement is a major predictor for local disease recurrence. Other prognostic markers include tumor size, number of mitoses, and presence of stromal atypia [6]. Some authors have reported that optimal management of this should include a mastectomy as wide local excision seems to be complicated by local recurrence [7]. Axillary lymph node dissection has not been suggested in literature. In contrast, sarcomatoid carcinomas and primary breast sarcomas often require axillary node dissection. Benefits of adjuvant chemotherapy and radiotherapy in terms of overall survival advantage have not been established yet [8]. Indications for adjuvant chemotherapy and radiation therapy, in the absence of specific data on breast sarcoma, should follow those for soft tissue sarcomas in general [9]. Osteosarcomas are aggressive tumors with blood borne spread more common than lymphatic spread and commonly occur in the lungs $(80 \%)$, bone (20\%), and liver (17\%) [2]. For same reason, axillary lymph node dissection is not considered alone with primary surgery. Diagnosis of metaplastic carcinoma should be considered in the presence of lymph nodes metastases [5]. Cystosarcoma phyllodes and metaplastic carcinoma are the closest differential diagnoses for osteosarcoma of the breast adifferentiated by specific morphological and IHC features. [6].

There is little evidence on the long-term prognosis of the disease due to the small number of cases reported in the literature. Silver et al. in their study of 50 patients with primary breast osteosarcoma reported a 5-year survival of 38\%, with $28 \%$ of patients developing local recurrence and $41 \%$ with distant metastases [10].

Our patient was a 44-year-old woman diagnosed with right-sided metaplastic breast carcinoma on cytology. She underwent upper-inner quadrantectomy with complete axillary dissection. Final histopathology and IHC was consistent with primitive osteoblastic osteosarcoma. Adjuvant chemotherapy was given and is doing fine till 13 months of followup.

Acknowledgements Bombay Hospital
Contribution Details Prashant Balwant Kerkar contributed to the concepts, design, definition of intellectual content, literature search, clinical studies, manuscript preparation, manuscript review, and guarantor of the study. Garima Daga contributed to the concepts, definition of intellectual content, clinical studies, manuscript preparation, and manuscript editing of the study.

\section{Compliance with Ethical Standards}

Conflict of Interest The authors declare that they have no conflict of interest.

\section{References}

1. Khan S, Griffiths EA, Shah N, Ravi S (2008) Primary osteogenic sarcoma of the breast: a case report. Cases J 1:148

2. Dey S, Chaudhury MK, Basu SK, Manna AK, Dutta SK (2013) Primary osteosarcoma of breast, a rare case. J Clin Diagn Res 7: 1710

3. Zhao J, Zhang X, Liu J, Li J (2013) Primary osteosarcoma of the breast with abundant chondroid matrix and fibroblasts has a good prognosis: a case report and review of the literature. Oncol Lett 6: 745-747

4. Uner A, Ozturk B, Benekli M, Coskun U, Kocak S, Kaya AO, Unsal D, Erekul S, Yaman E, Yildiz R, Buyukberber S (2008) Synchronize primary breast osteosarcoma and contralateral benign cystosarcoma phylloides: radiologic and pathologic imaging. Breast J 14:109-110

5. Ogundiran TO, Ademola SA, Oluwatosin OM, Akang EE, Adebamowo CA (2006) Primary osteogenic sarcoma of the breast. World J Surg Oncol 4:90

6. Adem C, Reynolds C, Ingle JN, Nascimento AG (2004) Primary breast sarcoma: clinicopathologic series from the Mayo Clinic and review of the literature. Br J Cancer 91:237-241

7. Uccello M, Malaguarnera M, Giordano M, Leggio E, Catania VE, Consoli AS, Trainiti M (2012) A large calcified retroperitoneal extraskeletal osteosarcoma with consequent bilateral hydronephrosis. Eur Rev Med Pharmacol Sci 16:977-982

8. Momoi H, Wada Y, Sarumaru S, Tamaki N, Gomi T, Kanaya S, Katayama T, Ootoshi M, Fukumoto M (2004) Primary osteosarcoma of the breast. Breast Cancer 11:396-400

9. Barrow BJ, Janjan NA, Gutman H, Benjamin RS, Allen P, Romsdahl MM, Ross MI, Pollock RE (1999) Role of radiotherapy in sarcoma of the breast-a retrospective review of the MD Anderson experience. Radiother Oncol 52:173-178

10. Silver SA, Tavassoli FA (1998) Primary osteogenic sarcoma of the breast: a clinicopathologic analysis of 50 cases. Am J Surg Pathol 22:925-933 